\title{
Criminal tendency detection from facial images and the gender bias effect
}

\author{
Mahdi Hashemi ${ }^{1 *}$ [C and Margeret Hall ${ }^{2}$
}

${ }^{*}$ Correspondence:

mhashem2@gmu.edu

${ }^{1}$ Department of Information

Sciences and Technology,

George Mason University,

4400 University Dr, Fairfax, VA 22030, USA

Full list of author information is available at the end of the article

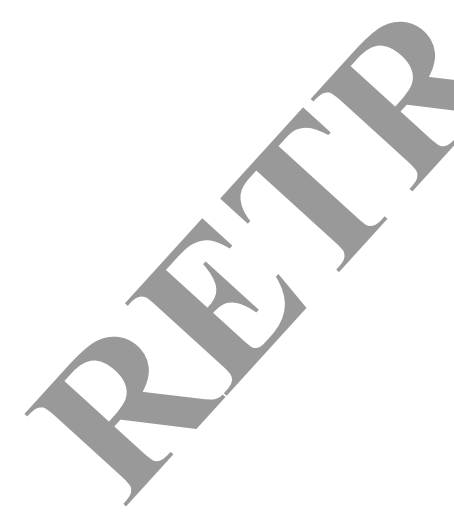

\section{Introduction}

Face is the primary means of recognizing a person, transmitting information, communicating with others, and inferring people's feelings, among others. Our faces might disclose more than what we expect. A facial image can be informative of personal traits [1], such as race, gender, age, health, emotion, psychology, and profession.

This study is triggered by Lombroso's research [2], which showed that criminals could be identified by their facial structure and emotions. While Lombroso's study looked at this issue from a physiology and psychiatry perspective, our study investigates whether or not machine learning algorithms would be able to learn and distinguish between criminal and non-criminal facial images. More specifically, we will look for gender biases in machine predictions. This is important because criminal facial images used to train 
the machine are mostly male. This is the result of the large gap between the number of mugshots for arrested males and females, available to the public and used to train the machine.

It is noteworthy that this study's scope is limited to the technical and analytical aspects of this topic, while its social implications require more scrutiny and its practical appli cations demand even higher levels of caution and suspicion. With that in mind, this paper explores the deep learning's capability in distinguishing between crimir and non-criminal facial images. To this effect, two deep learning models, a standar' feec ward neural network (SNN) and a convolutional neural network $(\mathrm{CNN})$, are trained wit, 10,000 neutral-emotion, mixed-gender, mixed-race facial images. A neutral blank face expression is characterized by neutral positioning of the facial feature neutral face expression could be caused by a lack of emotion, boredom, depr sion, or ght confusion. A neutral face expression is also referred to as a poker ace. is meant to conceal one's emotions when playing the card game poker [3\% ile bot, neural network models are controlled for facial emotions by applying on ne emotion images, no control has been imposed on race, due to our small dataset a the difficulty and occasionally subjectivity of identifying the race from low 1 are trained with and without controlling for gender. The results indicated that controlling for gender does not have much effect on accura $y$ or learning and both models reach high classification accuracies rege dless. $\mathrm{NN}$ achieves a tenfold cross-validation accuracy of $97 \%$.

The strength of this study lies i its applic, con of neural networks to investigate if a stack of non-linear functions vit. ousa ds of parameters can find useful facial features to distinguish betweer riminal a a non-criminal face shots. Its weakness however lies in its reliance on maciine arn these features and on a limited number of images.

"Related work" ser ion provides a review of related works. "Methodology" section elaborates on this s dy's me hodology. "Data preparation" section describes the image dataset sources and $t$. proach taken to prepare the dataset. "Neural network architecture" section ihes the SNN's and CNN's architecture, proposed in this study, for criminal tonden $y$ recognition through facial images. "Visual criminal tendency detection $r$ sult and d.scussion" section presents the results for both mixed gender and male or. $\mathrm{x}$ cla "fication scenarios. "Conclusion and future directions" section concludes the ver by $d_{1,}$,cussing the results and future directions.

\section{Related work}

$\mathrm{N}$ achine learning has shown to be more effective than humans in discovering personality traits through facial images [4]. Geng et al. [5] trained a machine to estimate the age through facial images. Reece and Danforth [6] applied an ensemble of machine learning models and image processing to detect depression and psychiatric disorder in Instagram facial images.

The goal in facial emotion detection is to train a machine to distinguish among six emotional facial expressions: happiness, surprise, sadness, disgust, anger, and fear [7]. Fuzzy inference system [8], hidden Markov model based on real-time tracking of the mouth shape [9], and Bayesian network [10] are among the approaches used for classifying facial emotions. 
Criminal tendency is another personality trait. Lombroso [2] was the first in 1871 to point out that criminals could be identified by their facial structure and emotions. Recently, Wu and Zhang [11] revisited this theory and quantitatively demonstrated the correlation between criminality and facial features. They trained four classifiers: logistic regression, $\mathrm{k}$ nearest neighbors (KNN), support vector machines (SVM), and convoly. tional neural network $(\mathrm{CNN})$ and claimed that their machine can identify a criminal face with a $90 \%$ accuracy. Their model was controlled for gender, race, and facial exp sion of emotions.

Neural networks have resurged and drawn much attention in the last decade [12] with the new brand of deep learning, mainly due to the significant perforn ce gain in visual recognition tasks [13]. Deep learning has been applied to a via nnge of applications, such as tree disease recognition [14]. Among the most "evant ap, acations of deep learning to our work, we can point to the application of $\mathrm{C} \wedge$ for face recognition [15, 16]. Cristani [17] and Segalin et al. [18, 19] applie th thine learning to predict the self-assessed personality traits (openness to experic ientiousness, extraversion, agreeableness, and neuroticism) of a person from images he/she uploads or likes on social media, such as Flicker, and what ins in terms of personality traits those images trigger in unacquainted people. They performed their experiments with 60,000 images from 300 Flickr users. Cristani et al. [17] and Segalin et al. [18] used a hybrid approach where generative $\mathrm{m}$ dels, $\mathrm{d}$ as latent representations of features (color, composition, textural properties, $\mathrm{c}$ ex racted from images, are built and then passed to a discriminative classif $r$ to predio each user's personality traits. Simplifying the problem into five distinct ina. lass acation problems, one for each trait, Segalin et al. [19] applied AlexNet ol, which, s an eight-layer version of CNN, pre-trained on ImageNet 2012 competition $a_{c}$-et. The problem they pose is to detect the personality traits based on th images than one uploads or likes on social media, such as Flicker. Their results showe hat the personality trait that others attribute to a person (based on the images that that in aual uploads or likes on social media) can be predicted $10 \%$ more accuratel the personality traits that that individual attributes to him/her-self. Wang an- Kosin ki [4] trained a deep neural network to classify facial images based on sexur orie tation.

\section{1. thodology}

Figu. $y$ shows the general workflow of this study. The first step is data collection and preparation which is performed as follows.

\section{Data preparation}

A total of 8401 gray-scale mugshot images of arrested individuals are obtained from National Institute of Standards and Technology (NIST) Special Database [20]. Images are all in png format. Images are of mixed race, mixed gender, and neutral face expression and contain both front and side (profile) views. Since our focus is on frontal face shots, we need to eliminate profile views. Haar feature-based cascade classifier [21] detects images containing frontal face views and also detects the rectangular area containing the face. Images are passed to the pre-trained version of this classifier, available in the OPenCV library in Python, to keep only the images that contain frontal face views 


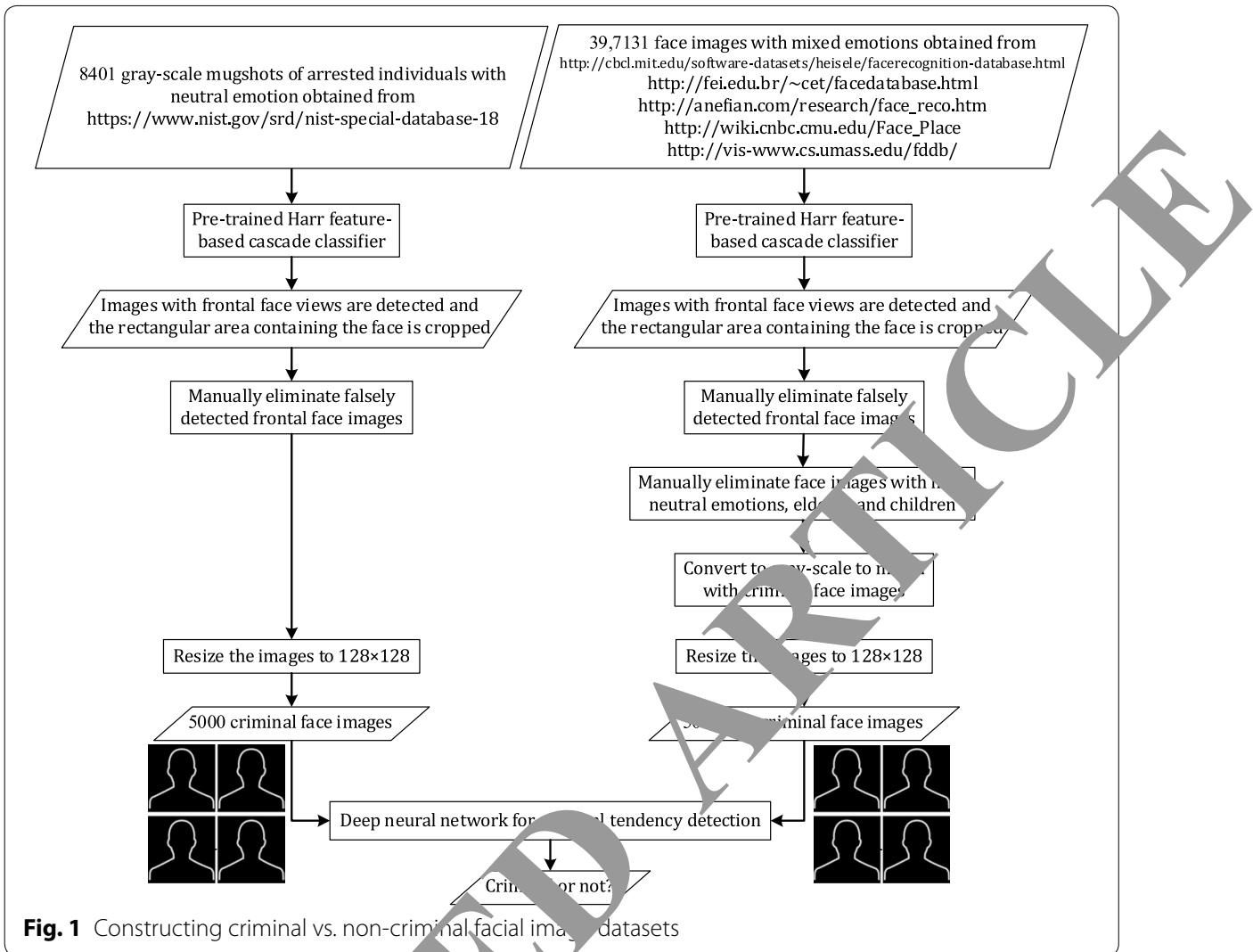

and then crop the rectangula vea containing the face. Cropping the facial rectangle from the rest of the mage preve,its the classifier from being affected by peripheral or background effects urrounding the face. The false positive rate (none-frontal face images misclassified a cal face images) of the Haar feature-based cascade classifier was $1.9 \%$ whic manually deleted. The result contains 5000 front view face images of 4796 male an 1204 female individuals and of variable sizes, ranging from $238 \times 238$ up to $13,813 \mathrm{p}$-xels. Since neural networks receive inputs of the same size, all images ar resi. to $128 \times 128$ using bilinear interpolation. This size is chosen considering the vacity o our platform (64-bit $3.00 \mathrm{GHz}$ Xeon E3-1505 M v6 processor, $2400 \mathrm{MHz}$ 16 DDR4 SODIMM RAM, NVIDIA Quadro M2200 4 GB GDDR5 GPU) to process the images collectively.

A total of 39,713 RGB facial images are obtained from five sources (Face Recognition Database [22], FEI Face Database [23], Georgia Tech face database [24], Face Place [25], Face Detection Data Set and Benchmark Home [26]). We consider these images as noncriminal face shots. Images are all in jpg format. Images are of mixed race, mixed gender, and mixed facial expressions. The database contains both front and side (profile) views. Since our focus is on frontal face shots, we need to eliminate profile views, using the Haar feature-based cascade classifier [21]. The false positive rate (none-frontal face images misclassified as frontal face images) of the Haar feature-based cascade classifier was $1.3 \%$ which were manually deleted. Facial images with any emotion expression but neutral are manually deleted, in order for compatibility with criminal facial images 
which are all neutral. Also, to keep the age, approximately, in the same range with the criminal dataset, images of elderly and children are manually deleted from this dataset. The images are then converted to gray-scale, again to be compatible with mugshots in the criminal dataset. The result contains 5000 front view face images of 3727 male and 1273 female individuals and of variable size, ranging from $87 \times 87$ up to $799 \times 799$ pixels Images are resized to $128 \times 128$.

\section{Neural network architecture}

As shown in Fig. 1, the data are passed to an artificial neural network for urther classification. Artificial neural networks do not rely on hand-engineer fea shich are hard to select and design. The neural network in our application rec es, as input, $128 \times 128$ pixel gray-scale images. Without extra preprocessir.s he image pixels are only divided by 255 so that they are in the range 0 to 1 . Before describ the neural network architecture, we justify our choice of activation fun tion loss function, and training algorithm.

While saturated activation functions, e.g. sigmoid $r$ tanh, cy ald trigger the vanishing gradients problem and prevent the exploding gradients py olem because of their nearzero gradient at large values, non-saturated activation iunctions, e.g. rectified linear unit (ReLU), could trigger the exploding gradi roblem and prevent the vanishing gradients problem because of their non-zer. vadiel at large values. Both problems happen for synaptic weights at lower layer and $w$ nrevent the network from being properly trained. The exploding gradient $\mathrm{bb} \cdot \mathrm{m}$ 's easier to detect because the vanishing gradients could also happen dur to the aining convergence. Besides, non-saturated activation functions make the ning several times faster [13]. Therefore, we chose the non-saturated activatic. functio $\operatorname{ReLU}[27,28]$. ReLU is a piecewise linear function, defined as the posit e part of its argument: $\operatorname{ReLU}(z)=\max (0, z)$. By projecting negative inputs to zero, ReLl reates a sparseness in the activation of neural units, a desirable effect similar dropout. The softmax function, $\operatorname{softmax}\left(z_{i}\right)=\exp \left(z_{i}\right) / \Sigma_{j} \exp \left(z_{j}\right)$, used in the final layer, taron $\mathrm{ms}$ the values $\left(z_{i}\right)$ to normalized exponential probabilities whose summan is on (i.e. $\left.\Sigma_{c} p_{c}=1\right)$. This provision $\left(\Sigma_{c} p_{c}=1\right)$ is a prerequisite for the application $f_{c}$ ntropy loss function, which is calculated as: $-\Sigma_{c} y_{c} \log \left(p_{c}\right)$, where $c$ repesents a yron (or class) in the output layer, $y_{c}$ represents the desired value (0 or 1$)$ at th. neuron, and $p_{c}$ is the predicted probability at that neuron. The cross-entropy loss funct on simplifies to $-(y \log (p)+(1-y) \log (1-p))$ for the binary classification in our clse. The network is trained using the Adam optimization algorithm [29], which is an extension to the stochastic gradient descent (SGD) approach, with a batch size of 100 . Unlike SGD which maintains a single and fixed learning rate for all synaptic weight updates, Adam continually adjusts individual adaptive learning rates for each synaptic weight based on estimates of first and second moments of the gradients. The learning rate is initialized at 0.0001 and the exponential decay rate for the first and second moment estimates are set to 0.9 and 0.999 respectively, suggested by Kingma and Ba [29].

Two neural network architectures are applied for classifying facial images into criminal and non-criminal categories, an SNN and a CNN. The SNN composes of four fully-connected layers, in addition to the input layer which has 16,384 neurons, 
equal to the total number of pixels in an image. The first three fully-connected layers have 512 neurons and each are followed by an ReLU layer. The fourth fully-connected layer has a size equal to the number of target categories, labeled as criminal and noncriminal, and is followed by a softmax function. The overall architecture is shown in Fig. 2.

$\mathrm{CNN}$ has recently outperformed other neural network architectures and other machine learning and image processing approaches in image classification [1 30 36] and object detection [37] due to its independence from hand-crafted y-ual tures and excellent abstract and semantic abilities [34,38]. CNN make strong and mostly correct assumptions about the nature of images, namely, yoca of pixel dependencies and stationarity of statistics. Therefore, in comparicon th SNN with similarly-sized layers, $\mathrm{CNN}$ has much fewer connections aram ers which makes it easier to train. The applied CNN in this work composes o vo convolutional layers followed by two fully-connected layers. Convoluticha hyers he the following settings: $f_{1}=3 \times 3 \times 1, s_{1}=1, n_{1}=8, f_{2}=3 \times 3 \times 8, s_{2}=1$, where $f_{m}, s_{m}$, and $n_{m}$ denote the size, stride, and number of filters of the $m$-th lay a vespectively. Every convolutional layer is followed by a max pooling and $R_{1}$ ror. Pooling summarizes the outputs of neighboring groups of neurons in the san kernel map. We use $2 \times 2$ max pooling with a stride of 2 , which means the pooling Kegions do not overlap. Smaller pooling regions cause over-fitting (hig varm e) and larger regions are too generic and lose the details (high bias [391). Th. Grst fully-connected layer has 64 neurons

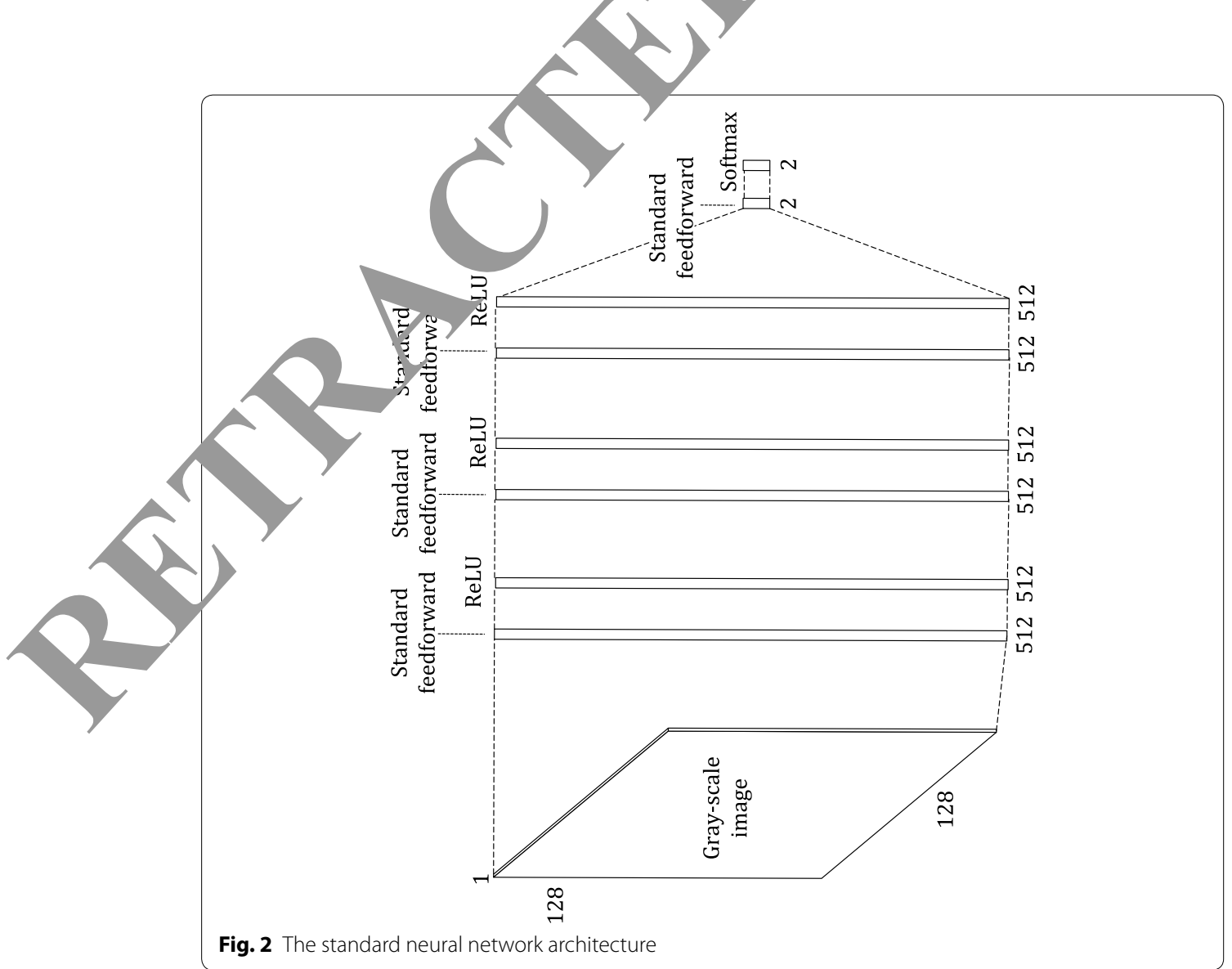


and is followed by an ReLU layer. The second fully connected layer has a size equal to the number of target categories, labeled as criminal and non-criminal, and is followed by a softmax function. The overall architecture is shown in Fig. 3.

The convolution filter and the pooling filter (elaborated in the next section) would slip outside the input image into the void, when they attempt to center themselves at bordep ing pixels. There are two strategies to solve this issue: (a) stopping the filter before it slips outside the image and (b) padding the input image with zero pixels. The first an oach

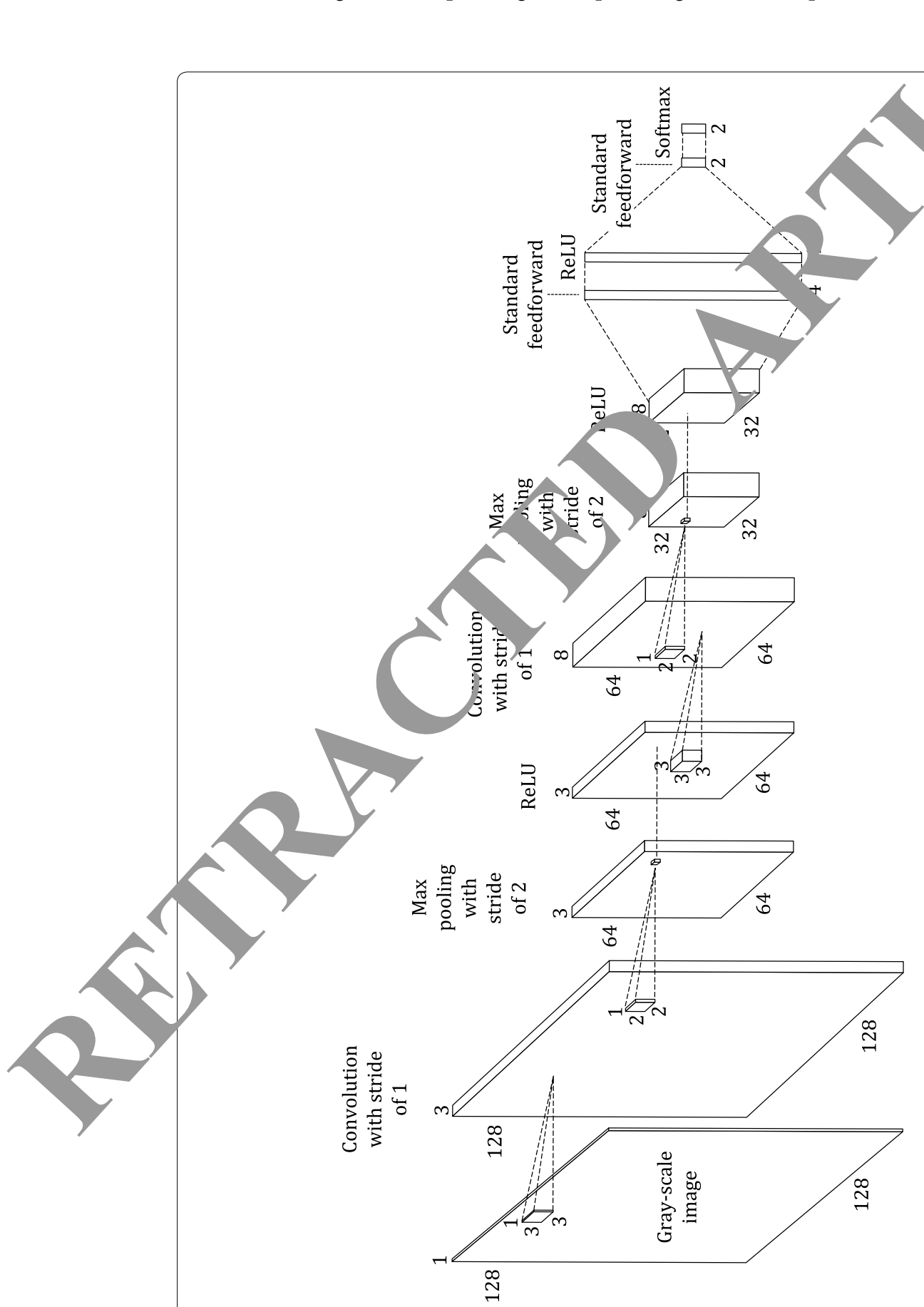

Fig. 3 The convolutional neural network architecture 
comes at the cost of under-scanning the bordering pixels because the filter will not get a chance to center itself at the bordering pixels. The second approach is referred to as padding and is the one applied in our model.

\section{Visual criminal tendency detection results and discussion}

Experiments are conducted on a 64-bit $3.00 \mathrm{GHz}$ Xeon E3-1505M v6 pro sssor, 2400 MHz 16 GB DDR4 SODIMM RAM, NVIDIA Quadro M2200 4 GB GDD R5 Artificial neural network models are implemented in Python using the TensorFloy, library [40].

Splitting a small dataset into training and testing sets would leavo wim even a smaller training set. In cross-validation, all the samples could be ised for $\mathrm{h}$ training and testing, while the model is evaluated on previously unse $n$ sa les. Additionally, in $\mathrm{k}$-fold cross-validation, we train and test $\mathrm{k}$ models. Thi - ws us $\mathrm{w}$ be more confident in the performance results. Consequently, we can $h$ on ort a more solid test accuracy, but also the standard deviation for this test accura Finally, cross-validation allows us to tune the number of layers in our neural i which will be further elaborated at the end of this section. With these advantage in mind, the tenfold cross-validation approach is applied here. The tenfold is preferre, over its fivefold counterpart to produce a more accurate standard devia ion.

The neural networks are trained un to $\urcorner$ ep chs, after which the change in training accuracy becomes imperceptible the charts $A$ Fig. 4 represent the average and standard deviation of training and tost a raci s at each epoch. The tenfold cross-validation has been performed at each noch. Th, the training and test accuracies at each epoch, reported in Fig. 4, are the vera, over the ten folds. The standard deviation of accuracies is also calculated ove the ten fold, at each epoch and depicted using the line's thickness. The CNN achieves s highest test accuracy (97\% with a standard deviation of $0.91 \%$ ) at epoch 306. While Aning accuracy keeps rising after this epoch, the test accuracy starts dro The test accuracy of 97\%, achieved by CNN (Fig. 4a), exceeds our expectations and is a clear indicator of the possibility to differentiate between criminals and $\mathrm{n} n$-c minal, using their facial images. It is noteworthy that the criminal mugshots ar con trom a different source than non-criminal face shots. That means the condins unde, which the criminal images are taken are different than those of non-criminal ages. These different conditions refer to the camera, illumination, angle, distance, background, resolution, etc. Such disparities which are not related to facial structure, th Jugh negligible in majority of cases, might have slightly contributed in training the classifier and helping the classifier to distinguish between the two categories. Therefore, it would be too ambitious to claim that this accuracy is easily generalizable.

Interestingly but not surprisingly, the CNN (Fig. 4a) achieves a higher test accuracy than the SNN (Fig. 4b), also in a more consistent way. The CNN's best test accuracy (97\%) is $8 \%$ higher than the SNN's best test accuracy (89\% with a standard deviation of $1.18 \%$ ). This goes back to the SNN being general purpose but the CNN being specifically designed for image classification. On the other hand, the training accuracy is only $0.37 \%$ different for $\mathrm{CNN}$ and $\mathrm{SNN}$, pointing to their equal capacity in learning from the training data. The $\mathrm{CNN}$ is more consistent in learning because the variance around its 

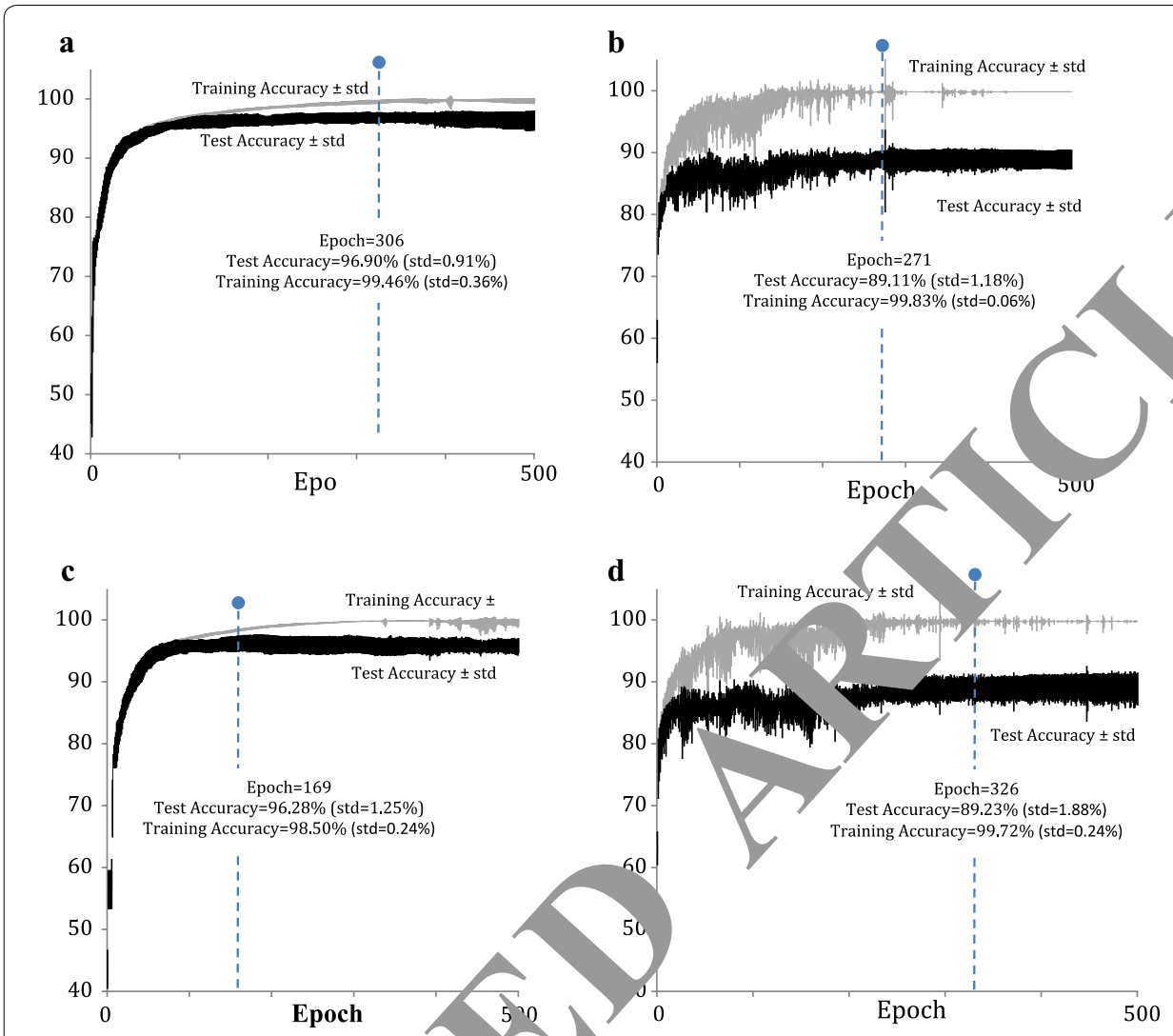

Fig. 4 Training and test accuracy wit one dard deviation of uncertainty at different epochs for: $\mathbf{a} C N N, \mathbf{b}$ SNN, c CNN when applied to only ale images, d d SNN when applied to only male images

training and test ace racy curves (Fig. 4a) is tighter than that of the SNN (Fig. 4b). The higher consistency a ccu acy of the $\mathrm{CNN}$ are because of its assumption of locality of pixel depender and its fewer parameters.

The confusion raty 1 xes for the CNN and SNN are shown in Tables 1 and 2, respectively. The liffere -ce between the false positive and false negative rates is $1 \%$ for the

Tab 1 Confusion matrix for CNN

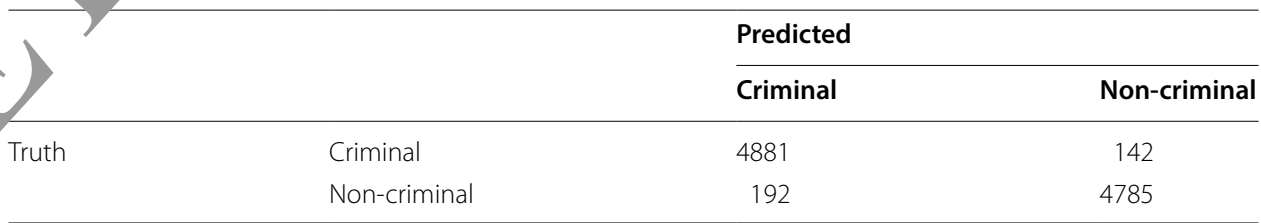

Table 2 Confusion matrix for SNN

\begin{tabular}{lllc}
\hline & & Predicted & \\
\cline { 3 - 4 } & & Criminal & Non-criminal \\
\hline Truth & Criminal & 4515 & 508 \\
& Non-criminal & 604 & 4373 \\
\hline
\end{tabular}


Table 3 Confusion matrix for CNN when applied to only male images

\begin{tabular}{|c|c|c|c|}
\hline & & \multicolumn{2}{|l|}{ Predicted } \\
\hline & & Criminal & Non-criminal \\
\hline \multirow[t]{2}{*}{ Truth } & Criminal & 4694 & 116 \\
\hline & Non-criminal & 261 & 3452 \\
\hline \multirow{2}{*}{\multicolumn{4}{|c|}{ Criminal }} \\
\hline & & & \\
\hline \multirow[t]{2}{*}{ Truth } & Criminal & 4423 & \\
\hline & Non-criminal & 555 & 8 \\
\hline
\end{tabular}

$\mathrm{CNN}$ and $2 \%$ for the SNN. In other words, the false posit. almost the same for both $\mathrm{CNN}$ and $\mathrm{SNN}$, i.e. the classifier no meaningful bias in making either type of mistake more than the other. $\mathrm{Ne}_{\mathrm{C}}$ sserved that there are misclassified men, women, white, and colored people from both categories. Among the false negatives (criminal images which were misclncsified as non-criminal) by $\mathrm{CNN}, 81 \%$ were male and $19 \%$ were female. This is pro rtionc o the $75 \%$ male vs. $25 \%$ female images among non-criminals. Among the f ${ }^{\prime} \mathrm{se} p \mathrm{po}$ ive (non-criminal images which were misclassified as criminal) by $\mathrm{CNN}, 8 \%$ " wore mal, and $12 \%$ were female. This is proportional to the $95 \%$ male vs. $5 \%$ femal ima an ang criminals. Among the false negatives by $\mathrm{CNN}, 79 \%$ were white peop and $21 \%$ were colored people. This is proportional to the $69 \%$ white vs. $31 \%$ colored peo CNN, $79 \%$ were whi e and $21 \%$ were colored. This is proportional to the $72 \%$ white vs. $28 \%$ colored people nong criminals. This indicates that the classifier is not biased to put people of a specific onder or race in a specific category while ignoring their criminal tendency.

There mor females among non-criminal images than criminal ones. While $25 \%$ of no cri : inol i, nages are female, only $4 \%$ of criminal images are female. The machine might bu nfairly taking advantage of this distinction to boost its classification accuracy.

observe and control the gender bias effect, we separate male and female images in each tegory. Since the number of female images is too small, we only train and crossvalidate the models using male images. There are 4796 male images in the criminal and 3/27 in the non-criminal category. Figure 4c, d show the average and standard deviation of training and test accuracies over different training epochs for the CNN and SNN, respectively. These charts very closely imitate their mixed gender counterparts in Fig. 4a, $\mathrm{b}$, a sign that gender has no effect on biasing the classifier one way or the other. The corresponding confusion matrixes for $\mathrm{CNN}$ and $\mathrm{SNN}$ when applied to only male images, shown in Tables 3 and 4, endorse the same conclusion.

Choosing the $\mathrm{CNN}$ to have two convolutional layers was the result of an experimental model complexity vs. generalization accuracy analysis. Figure 5 shows how changing the number of convolutional layers affects the tenfold cross-validation accuracy and its standard deviation. According to this graph, the CNN with five convolutional 


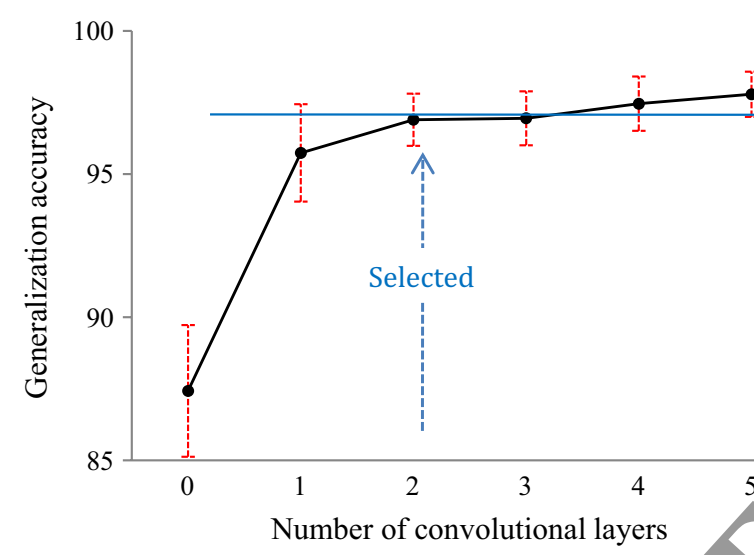

Fig. 5 Number of convolutional layers in CNN vs. tenfold cross-validation accur cy tits stancard deviation

layers achieves the highest accuracy. However, the accura volutional layers falls within one standard deviation margin os Me accuracy of the $\mathrm{CNN}$ with five convolutional layers. This is true for CNNs w. ree and two convolutional layers as well. Thus, the CNN with two convolution layers is considered optimum, in this case. The architecture of the $\mathrm{CNN}$ w.th two convolutional layers is explained in Sect. 3. The CNNs with less than two $c$ volut al layers are obtained by dropping the last convolutional layers. For $\mathrm{CNN}$ s with re han two convolutional layers, we have: $f_{3}=3 \times 3 \times 16, s_{3}=1, n_{3}=32, f \quad 3 \times 3 \times 32, s_{4}=1, n_{4}=64$ and $f_{5}=3 \times 3 \times 64, s_{5}=1$, $n_{5}=128$, where $f_{m}, s_{m}$, and $n_{n}$ den the size, stride, and number of filters of the $m$-th convolutional layer.

Facial features and $\mathrm{cr}$ minal tendency

Convolutional layers $\mathrm{CNN}$ are essentially feature generation layers. If a $\mathrm{CNN}$ achieves a high accurad it means that the generated features by convolutional layers are effective in distinguishin $\mathrm{b} / \mathrm{cr}$ en classes. Therefore, to understand what facial features are used by $\mathrm{CN}$ lassiv the images, we need to look at the facial features that are emphasized or pin in each convolutional layer. A convolutional layer usually has multiple filt rs. Each " ter separately contributes in feature generation, though it is their cumulative k. vledge that helps $\mathrm{CNN}$ to classify the images. Our $\mathrm{CNN}$ contains 2 convolutional layers the first one has 8 filters and the second one has 16 .

In Fig. 6, the output of one of the filters from the first convolutional layer and one of the filters from the second convolutional layer are visualized. They highlight the facial characteristics that are learned and used by $\mathrm{CNN}$ to distinguish between the two classes. Additionally, Fig. 6 compares these facial features between a criminal and non-criminal face shot. It is noteworthy that neither these facial features nor their differences are hard coded into the machine. They are learned by the machine as most helpful in classifying the two sets of images in the training dataset. Both convolutional layers detect and underscore the shape of the face, eyebrows, top of the eye, pupils, nostrils, and lips. 

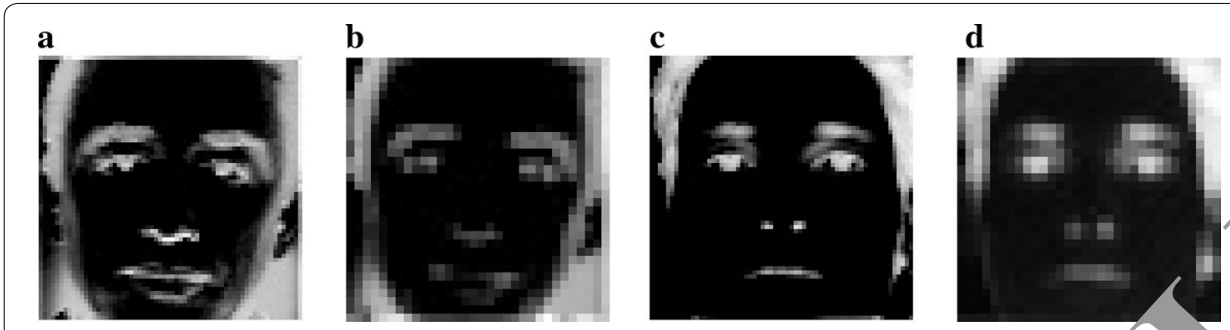

Fig. 6 Facial features detected by the first $(\mathbf{a}, \mathbf{c})$ and second $(\mathbf{b}, \mathbf{d})$ convolutional layers in $C N N$, for a - rim $(\mathbf{a}, \mathbf{b})$ vs. non-criminal (c, d) face shot

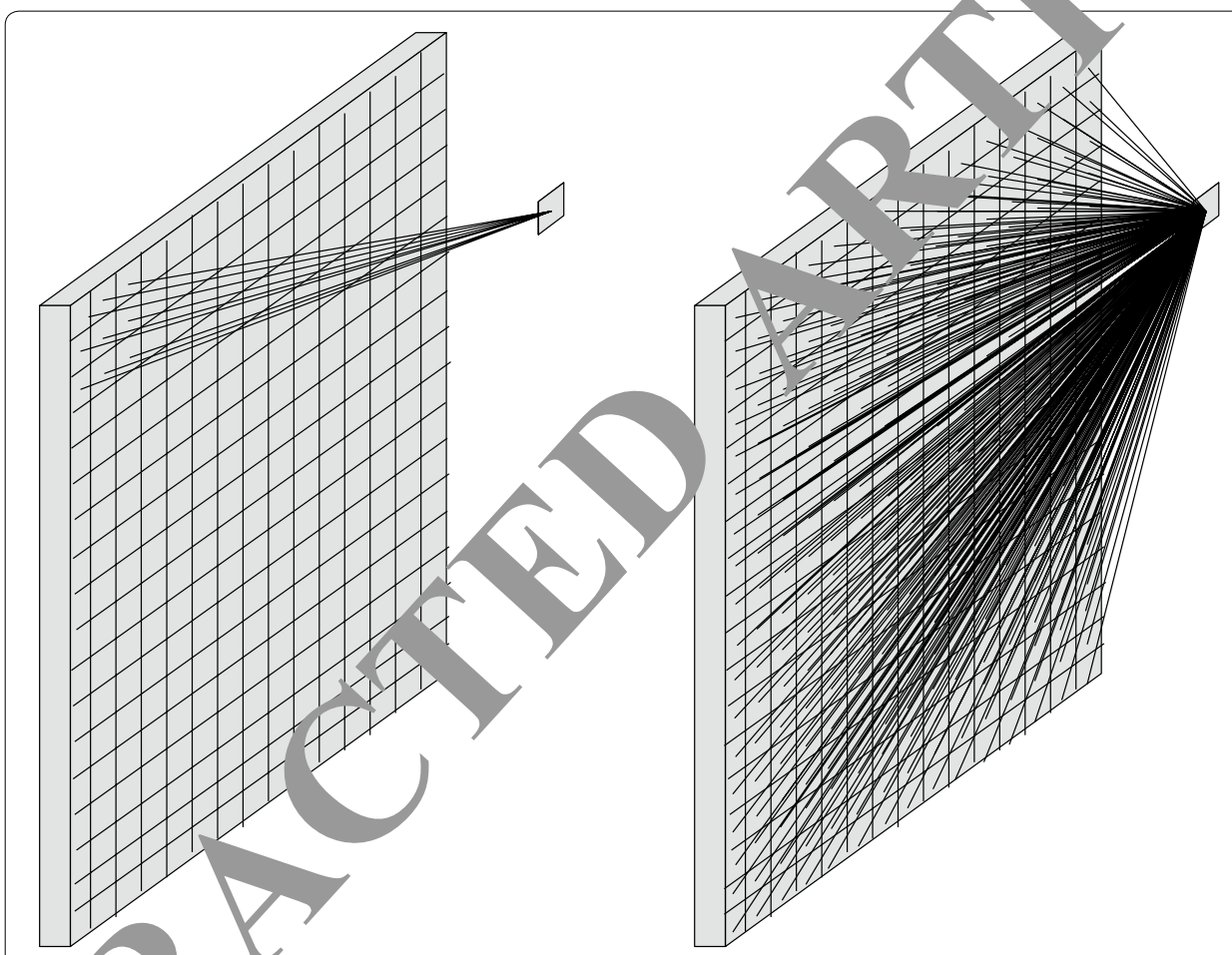

Fig. A no e in the hrst hidden layer is connected to only a small number of image pixels in CNN (left) while itis co teu cuall image pixels in SNN (right)

\section{Why $\mathrm{C}, \mathrm{NN}$ achieves higher accuracy than SNN?}

T) o architectural features of CNNs making them more convincing than SNNs for image classification are as follows:

a. Partial connectivity rather than full connectivity

A node in a $\mathrm{CNN}$ is connected only to a small number of nodes in the previous layer, while the same node in an SNN is connected to all nodes in the previous layer. This means that the number of synaptic weights that need to be calculated is mush fewer in $\mathrm{CNN}$ than SNN. Assume we use a $3 \times 3$ convolution window in the $\mathrm{CNN}$, shown on the left side of Fig. 7. This means a node in the first hidden layer, for instance, is only connected to 9 pixels in the image. The same node in the SNN, shown on the 
right side of Fig. 7, is connected to all the 270 pixels of the image. In other words, the number of synaptic weights is 30 times fewer in the CNN than SNN. Of course, this number depends on the size of both the image and convolution window. If the image is $n \times m$ and the convolution window is $z \times z$, the number of synaptic weights in CNN is $n \times m / z^{2}$ times fewer than SNN. We showed this only for the first hidde: layer, but the same is true for all convolutional hidden layers. This has two advantages. First, a much fewer unknown parameters (synaptic weights) can be rned more quickly (less computational complexity) and accurately by the mach:1.., w. significantly reduced chance of overfitting. Second, deriving the value of each node $\mathrm{i} / \mathrm{A}$ the next layer from only a small number of neighboring pixels, rather th the exitire image, is based on the assumption that the relationship between two "stant pixels is probably less significant than two close neighbors. This assun tion is in ared by the visual cortex system in humans and other animals.

b. Shared weights

We mentioned that $n \times m$ synaptic weights need to for one node in the first hidden layer of SNN. With $k$ nodes in the first hiddo ayer, a total of $n \times m \times k$ synaptic weights must be calculated, because eac... in the first hidden layer has its own synaptic weights which are different than those of other nodes. In a CNN, however, the number of synaptic weightc that need $\%$ be learned remains $z^{2}$, because nodes in the first hidden layer do p t hav lifferent synaptic weights, but share the same weights. Therefore, regardloss $\mathrm{O}_{\mathrm{a}} \mathrm{w} \mathrm{r}$ any nodes exist in the first hidden layer, the number of synaptic weir ts that ne, $a$ to be learned remains $z^{2}$. Consequently, the number of synaptic veigh in C $\mathrm{NN}$ is $n \times m \times k / z^{2}$ times fewer than SNN for

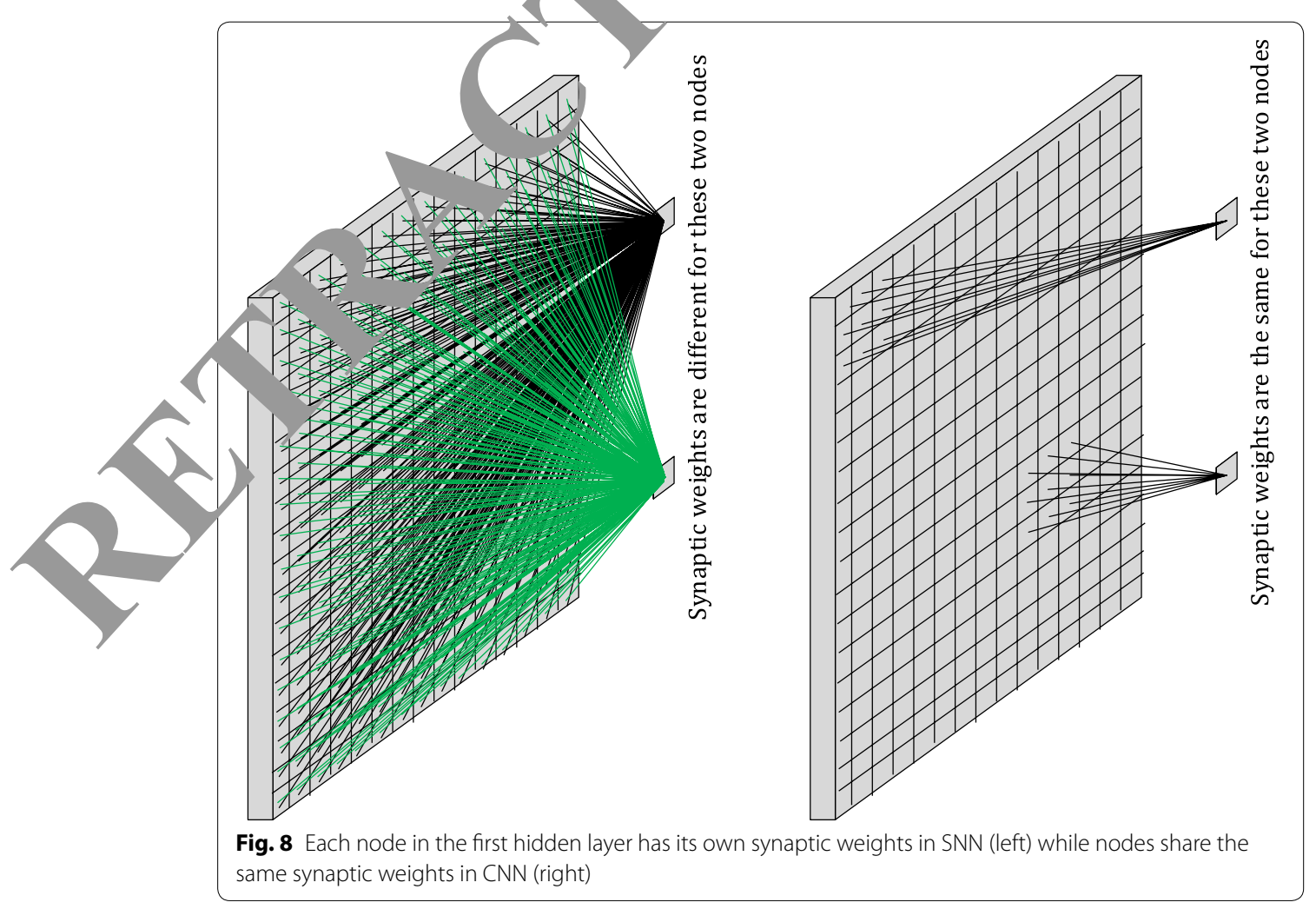


the first hidden layer. This is referred to as weight sharing property and is depicted in Fig. 8. Despite this explanation concerned the first hidden layer, it is true for all convolutional hidden layers. This property gives CNNs two advantages over SNN. The first advantage is even less parameters for the machine to learn and the second is enabling the $\mathrm{CNN}$ to look for certain objects in the image, regardless of where in th. image they are.

\section{Conclusions and future directions}

Classifying people in any manner requires care but predicting whethe a person is a criminal demands even more caution and scrutiny and must be locke rpon with suspicion. The danger of this technology lies in its imperfection sh misclassifying individuals can have grave repercussions. It would be too o imistic y claim that the $97 \%$ test accuracy, achieved by the CNN in this work, is eas generalizable to face shots from any other source. This is not only becaus of the small size of our dataset, but also the fact that criminal and non-crimina $m$ ome from different sources. Thus, the conditions under which the images are yen are not exactly the same, which raises the question, whether this disp as captured by the deep classifier to unfairly distinguis between the two classes. In an ideal dataset, all face shots, criminal and non-crimina, would be taken with the same camera and under the same condition i.e. hi nination, angle, distance, background, resolution, makeup, beard, hat, and glas

Facial emotions and age, mair sources,$y$ bias in classifying facial images based on criminal tendency, were cants od in our work by eliminating non-neutral facial images and images of eld and $\mathrm{ch}_{\mathrm{t}}$ dren. The bias due to background effects was mitigated by cropping the fac area out of images. The gender bias was not only eliminated by ignor ng female inages, but also measured and shown to be of little impact. Race, anotl $r$ source of bias, was not accounted for in this study because of our small dataset an difficulty and occasionally subjectivity of identifying the race from low facial images. However, both categories contain images of all races $w^{i^{\text {th }}}$ rougl $y$ similar proportions. Enlarging our dataset, measuring the impact of ra 91 b) c and detecting other personality traits form our future research venues.

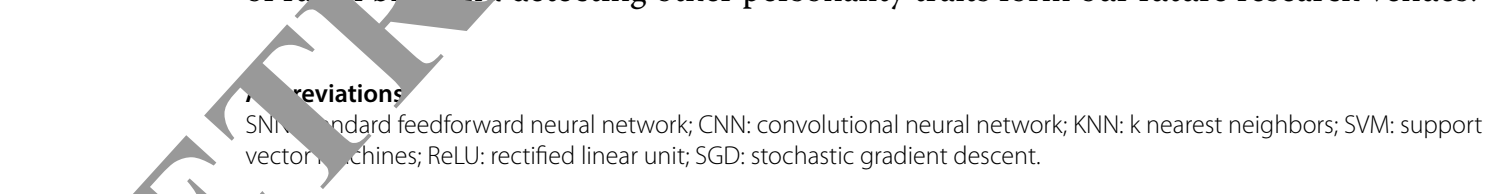

Acknowledgements

t applicable.

Authors' contributions

Both authors have contributed in developing the idea, documenting it, conducting the experiments, writing and revising. Both authors read and approved the final manuscript.

Funding

Not applicable.

Availability of data and materials

Not applicable.

Competing interests

The authors declare that they have no competing interests. 


\section{Author details}

${ }^{1}$ Department of Information Sciences and Technology, George Mason University, 4400 University Dr, Fairfax, VA 22030, USA. ${ }^{2}$ College of Information Science and Technology, University of Nebraska at Omaha, $1110 \mathrm{~S} 67$ th St, Omaha, NE 68182, USA.

Received: 31 August 2019 Accepted: 30 December 2019

Published online: 07 January 2020

\section{References}

1. Zebrowitz LA, Montepare JM. Social psychological face perception: why appearance matters. Soc Persol ar. Psyc, Compass. 2008;2(3):1497-517.

2. Lombroso C. Criminal man. 5th ed. Durham: Duke Univ. Press; 2006.

3. Hargrave J. Poker face: the art of analyzing poker tells. Dubuque: Kendall Hunt Pub Co; 2010.

4. Wang Y, Kosinski M. Deep neural networks are more accurate than humans at detecting sex rientur rom facial images. J Personal Soc Psychol. 2018;114(2):246-57.

5. Geng X, Yin C, Zhou ZH. Facial age estimation by learning from label distributions. IEF Trans Patten, Mal Mach Intell. 2013;35(10):2401-12.

6. Reece AG, Danforth CM. Instagram photos reveal predictive markers of depression.EPJ Da. i. 2017;6(1):15.

7. Ekman P, Friesen W. The facial action coding system (FACS): a technique for th asuremen of facial action. Palo Alto: Consulting Psychologists; 1978.

8. Tsapatsoulis N, Karpouzis K, Stamou G, Piat F, Kollias S. A fuzzy system for en ation based on the MPEG-4 facial definition parameter set. In: 10th European signal processing co ence; 2000. p. 1-4.

9. Oliver N, Pentland A, Bérard F. LAFTER: a real-time face and lips trac with facial spression recognition. Pattern Recogn. 2000;33(8):1369-82.

10. Zhang Y, Ji Q. Active and dynamic information fusion for facial expres. io ungerstanding from image sequences. IEEE Trans Pattern Anal Mach Intell. 2005;27(5):699-714.

11. Wu X, Zhang X. Automated inference on criminality usina face images. 2016. arXiv preprint. arXiv:1611.04135.

12. Taneja A, Arora A. Modeling user preferences using ueur. tworks and tensor factorization model. Int J Inf Manag. 2019;45:132-48.

13. Krizhevsky A, Sutskever I, Hinton GE. Imagenet clas, tion v th deep convolutional neural networks. In: Advances in neural information processing systems; 2012.p. 109,

14. Baranwal S, Khandelwal S, Arora A. Deep arning convoly, ional neural network for apple leaves disease detection. In: International conference on sustainab, nputin in science, technology \& management; 2019; Jaipur, India. p. 260-7.

15. Ouyang W, Wang X, Zeng X, Qiu P, Tian Y, L A, Yang S, Wang Z, Loy CC, et al. Deepid-net: deformable deep convolutional neural network for $\mathrm{o}_{\text {n }}$ detection. In: IEEE conference on computer vision and pattern recognition; 2015. p. 2403-12

16. Sun $Y$, Liang D, Wang $X$, ang $X$. Deepid3: ace recognition with very deep neural networks. 2015. arXiv preprint. arXiv:1502.00873.

17. Cristani M, Vinciarelli A, ralin C, P rina A. Unveiling the multimedia unconscious: Implicit cognitive processes and multimedia content anà,

18. Segalin C, Perii Cristani M, Vinciarelli A. The pictures we like are our image: continuous mapping of favorite pictures into sel -ass and attributed personality traits. IEEE Trans Affect Comput. 2017;8(2):268-85.

19. Segalin C, Cheng 8, Cristani M. Social profiling through image understanding: personality inference using convolution anth netwerks. Comput Vis Image Underst. 2017;156(1):34-50.

20. N' Spec I Databuse 18. 2010. https://www.nist.gov/srd/nist-special-database-18. Accessed 12 Apr 2019.

21. Vion ' nes mapid object detection using a boosted cascade of simple features. In: IEEE computer society confero on computer vision and pattern recognition; 2001. p. 1-9.

Face Reco, nition Database. http://cbcl.mit.edu/software-datasets/heisele/facerecognition-database.html. Accessed June 2018.

23. th Ce Database. http://fei.edu.br/ cet/facedatabase.html. Accessed 02 June 2018.

24. Georgia Tech face database. http://www.anefian.com/research/face_reco.htm. Accessed 02 June 2018.

2. Face Place. http://wiki.cnbc.cmu.edu/Face_Place. Accessed 02 June 2018.

Face Detection Data Set and Benchmark Home. http://vis-www.cs.umass.edu/fddb/. Accessed 02 June 2018.

27. Hinton GE, Srivastava N, Krizhevsky A, Sutskever I, Salakhutdinov RR. Improving neural networks by preventing coadaptation of feature detectors. 2012. arXiv preprint. arXiv:1207.0580.

28. Nair $V$, Hinton GE. Rectified linear units improve restricted boltzmann machines. In: The 27th international conference on machine learning; 2010. p. 807-14.

29. Kingma DP, Ba J. Adam: a method for stochastic optimization. 2014. arXiv preprint. arXiv:1412.6980.

30. Simonyan K, Zisserman A. Very deep convolutional networks for large-scale image recognition. 2014. arXiv preprint. arXiv:1409.1556.

31. Wang M, Liu X, Wu X. Visual classification by 11-hypergraph modeling. IEEE Trans Knowl Data Eng. 2015;27(9):2564-74

32. Yu J, Tao D, Wang M. Adaptive hypergraph learning and its application in image classification. IEEE Trans Image Process. 2012;21(7):3262-72.

33. Szegedy C, Liu W, Jia Y, Sermanet P, Reed S, Anguelov D, Erhan D, Vanhoucke V, Rabinovich A. Going deeper with convolutions. In: IEEE conference on computer vision and pattern recognition; 2015. p. 1-9. 
34. Zeiler MD, Fergus R. Visualizing and understanding convolutional networks. In: European conference on computer vision; 2014. p. 818-33.

35. Hashemi M, Hall M. Detecting and classifying online dark visual propaganda. Image Vis Comput. 2019;89(1):95-105.

36. Hashemi M. Enlarging smaller images before inputting into convolutional neural network: zero-padding vs. interpolation. J Big Data. 2019;6(1):98.

37. Farfade SS, Saberian MJ, Li LJ. Multi-view face detection using deep convolutional neural networks. In: 5th International conference on multimedia retrieval; 2015. p. 643-50.

38. Hashemi M. Web page classification: a survey of perspectives, gaps, and future directions. Multimedia Tools Appl. 2020. https://doi.org/10.1007/s11042-019-08373-8

39. Zeiler MD, Fergus R. Stochastic pooling for regularization of deep convolutional neural networks. 2013. arXiy oreprint. arXiv:1301.3557.

40. TensorFlow. TensorFlow Tutorials. 2019. https://www.tensorflow.org/tutorials. Accessed 01 Jan 2019.

\section{Publisher's Note}

Springer Nature remains neutral with regard to jurisdictional claims in published maps and instit / onal
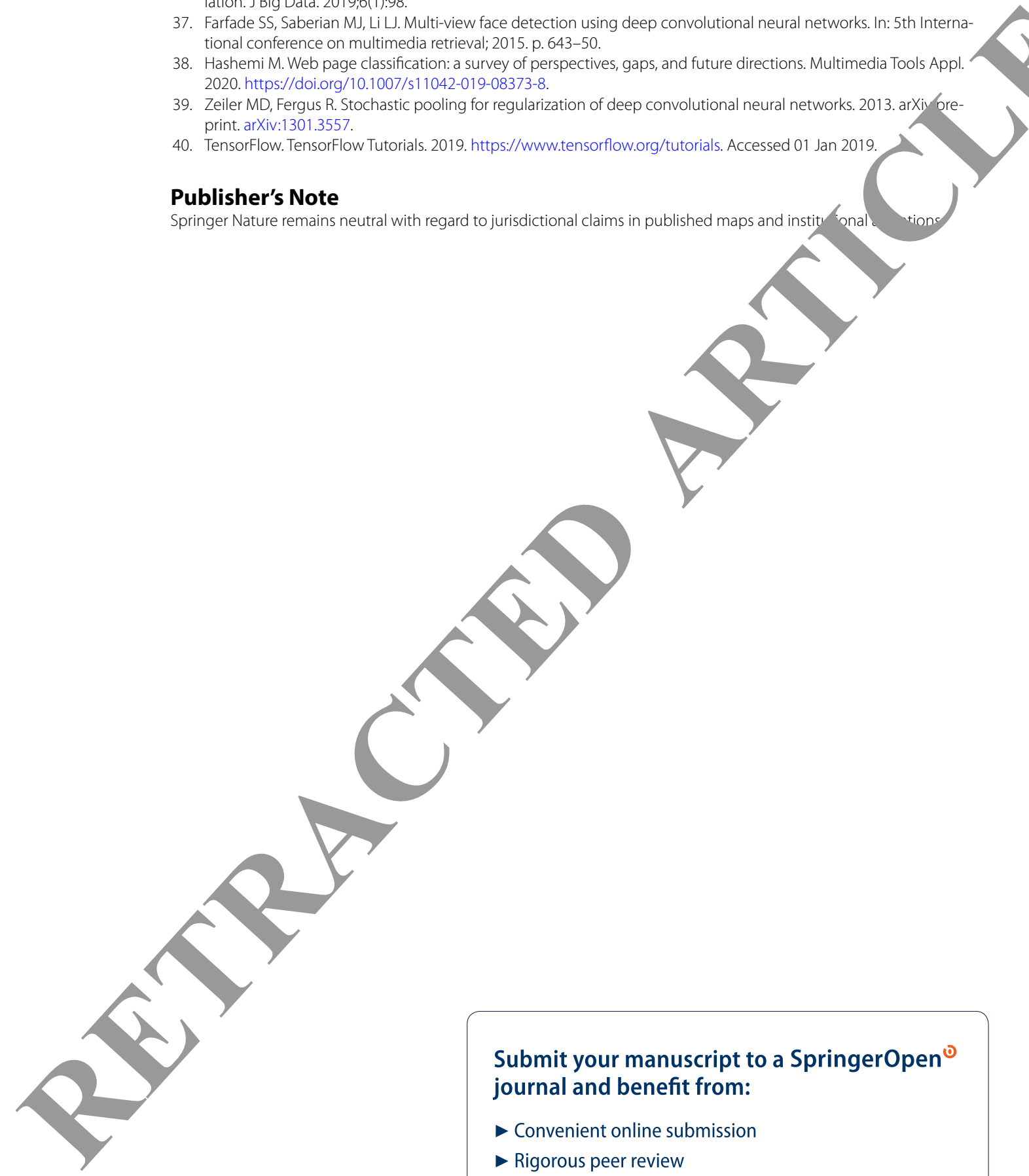